\title{
GRESHAM'S LAW OF MODEL AVERAGING
}

\author{
IN-KOO CHO AND KENNETH KASA
}

\begin{abstract}
An agent operating in a self-referential environment thinks the parameters of his model might be time-varying. In response, he estimates two models, one with time-varying parameters, and another with constant parameters. Forecasts are then based on a Bayesian Model Averaging strategy, which mixes forecasts from the two models. In reality, structural parameters are constant, but the (unknown) true model features expectational feedback, which the agent's reduced form models neglect. This feedback allows the agent's fears of parameter instability to be self-confirming. Within the context of a standard linear present value asset pricing model, we use the tools of large deviations theory to show that the agent's self-confirming beliefs about parameter instability exhibit Markov-switching dynamics between periods of tranquility and periods of instability. However, as feedback increases, the duration of the unstable state increases, and instability becomes the norm. Even though the constant parameter model would converge to the (constant parameter) Rational Expectations Equilibrium if considered in isolation, the mere presence of an unstable alternative drives it out of consideration.

JEL Classification Numbers: C63, D84
\end{abstract}

\section{INTRODUCTION}

Econometric model builders quickly discover their parameter estimates are unstable. ${ }^{1}$ It's not at all clear how to respond to this. Maybe this drift is signalling model misspecification. If so, then by appropriately adapting a model's specification, parameter drift should dissipate over time. Unfortunately, evidence suggests that drift persists even when models are adapted in response to the drift. Another possibility is that the underlying environment is inherently and exogenously nonstationary, so there is simply no hope of describing economic dynamics in models with constant parameters. Clearly, this is a rather negative prognosis. A third possibility is that instability reflects equilibrium indeterminacy and the presence of sunspots (Farmer $(1993,2010)$ ). Our paper considers a new possibility, one that is consistent with both the observed persistence of parameter drift, and its heteroskedastic nature. We show that in self-referential environments, where the agent's own beliefs influence the data-generating process (DGP), it is possible that persistent parameter drift becomes self-confirming. That is, parameters drift simply because agents think they might drift. We show that this instability can arise even in models that would have unique and determinate equilibria if parameters were known. Self-confirming

Date: February, 2015.

${ }^{1}$ See, e.g., Cogley and Sargent (2005), Fernandez-Villaverde and Rubio-Ramirez (2007), and Inoue and Rossi (2011) for evidence on parameter instability in macroeconomic models. Bacchetta and van Wincoop (2013) discuss parameter instability in exchange rate models. 
volatility arises here because agents are assumed to be unaware of their own influence over the DGP, and respond to it indirectly by adapting parameter estimates. ${ }^{2}$

\section{TO BELIEVE IS TO SEE}

To illustrate the basic idea, let us start with a motivating example. This example is inspired by the numerical simulations of Evans, Honkapohja, Sargent, and Williams (2013) using a cobweb model. We argue that the findings of Evans, Honkapohja, Sargent, and Williams (2013) can potentially apply to a broader class of dynamic models.

2.1. Motivating example. Consider the following workhorse asset-pricing model, in which an asset price at time $t, p_{t}$, is determined according to

$$
p_{t}=(1-\gamma) f_{t}+\gamma \mathrm{E}_{t} p_{t+1}+\epsilon_{p, t}
$$

where $f_{t}$ denotes economic fundamentals (e.g., dividends), and where $\gamma \in(0,1)$ is a (constant) discount rate, which determines the strength of expectational feedback. Empirically, it is close to one. The $\epsilon_{p, t}$ shock is Gaussian white noise. Fundamentals are assumed to be observed, and evolve according to the $\mathrm{AR}(1)$ process

$$
f_{t}=\rho f_{t-1}+\epsilon_{f, t}
$$

for $\rho \in(0,1)$. The fundamentals shock, $\epsilon_{f, t}$, is Gaussian white noise, and is assumed to be orthogonal to the price shock $\epsilon_{p, t}$. The unique stationary rational expectations equilibrium is

$$
p_{t}=\frac{1-\gamma}{1-\gamma \rho} f_{t}+\epsilon_{p, t}
$$

Along the equilibrium path, the dynamics of $p_{t}$ can only be explained by the dynamics of fundamentals, $f_{t}$. Any excess volatility of $p_{t}$ over the volatility of $f_{t}$ must be soaked-up by the exogenous shock $\epsilon_{p, t}$.

It is well known that Rational Expectations versions of this kind of model cannot explain observed asset price dynamics (Shiller (1989)). Not only are prices excessively volatile, but this volatility comes in recurrent 'waves'. Practitioners respond to this using reduced form ARCH models. Instead, we try to explain this persistent stochastic volatility by assuming that agents are engaged in a process of Bayesian learning. Of course, the notion that learning might help to explain asset price volatility is hardly new (see, e.g., Timmermann (1996) for an early and influential example). However, early examples were based on leastsquares learning, which exhibited asymptotic convergence to the Rational Expectations Equilibrium. This would be fine if volatility appeared to dissipate over time, but as noted earlier, there is no evidence for this. In response, a more recent literature has assumed that agents use so-called constant gain learning, which discounts old data. This keeps learning alive. For example, Benhabib and Dave (2014) show that constant gain learning can generate persistent excess volatility, and can explain why asset prices have fat-tailed distributions even when the distribution of fundamentals is thin-tailed.

Our paper builds on the work of Benhabib and Dave (2014). The key parameter in their analysis is the update gain. Not only do they assume it is bounded away from

\footnotetext{
${ }^{2}$ Another possibility is sometimes advanced, namely, that parameter drift is indicative of the Lucas Critique at work. This is an argument that Lucas (1976) himself made. However, as noted by Sargent (1999), the Lucas Critique (by itself) cannot explain parameter drift.
} 
zero, but they restrict it to be constant. Following Sargent and Williams (2005), they note that a constant gain can provide a good approximation to the (steady state) gain of an optimal Kalman filtering algorithm. However, they go on to show that the learning dynamics exhibit recurrent escapes from this steady state. This calls into question whether agents would in fact cling to a constant gain in the presence of such instability. Here we allow the agent to effectively employ a time-varying gain, which is not restricted to be nonzero. We do this by supposing that agents average between a constant gain and a decreasing/least-squares gain. Evolution of the model probability weights delivers a statedependent gain. In some respects, our analysis resembles the gain-switching algorithm of Marcet and Nicolini (2003). However, they require the agent to commit to one or the other, whereas we permit the agent to be a Bayesian, and average between the two. Despite the fact that our specification of the gain is somewhat different, like Benhabib and Dave (2014), we rely on the theory of large deviations to provide an analytical characterization of the Markov-switching escape dynamics.

2.2. Learning with a correct model. Suppose a decision maker has to learn a coefficient $b$. Let us assume that the decision maker is Bayesian, and has a Gaussian prior over $b$ with mean $b_{0}$. Let $\mathcal{M}_{0}$ be the model, which treats $b$ as an unknown constant, which must be learned. In order to invoke the Kalman filter, we need to re-write the right hand side of (2.1) in terms of observable variables at time $t$

$$
p_{t}=\frac{(1-\gamma) \rho}{1-\gamma \rho} f_{t-1}+\frac{1-\gamma}{1-\gamma \rho} \epsilon_{f, t}+\epsilon_{p, t} .
$$

For later reference, let us write $\mathcal{M}_{0}$ in a slightly more flexible form. Suppose that the decision maker believes $b$ is evolving over time according to $\left\{b_{t}\right\}$ where

$$
b_{t}=b_{t-1}+\epsilon_{b, t}
$$

and $\epsilon_{b, t}$ is i.i.d. Gaussian white noise with variance $\sigma_{b}^{2} . \mathcal{M}_{0}$ can be represented by a parametric restriction on $\sigma_{b}^{2}$ :

$$
\mathcal{M}_{0}: \sigma_{b}^{2}=0
$$

Note that $\mathcal{M}_{0}$ is correctly specified model, since the rational expectations equilibrium is in $\mathcal{M}_{0}$.

We write the perceived state space model as

$$
\begin{aligned}
& {\left[\begin{array}{l}
p_{t} \\
f_{t}
\end{array}\right] }=\left[\begin{array}{cc}
0 & f_{t-1} \\
\rho f_{t-1} & 0
\end{array}\right]\left[\begin{array}{c}
1 \\
b_{t}(i)
\end{array}\right]+\left[\begin{array}{c}
\frac{1-\gamma}{1-\gamma \rho} \epsilon_{f, t}+\epsilon_{p, t} \\
\epsilon_{f, t}
\end{array}\right] \\
& {\left[\begin{array}{c}
1 \\
b_{t+1}
\end{array}\right] }=\left[\begin{array}{ll}
1 & 0 \\
0 & 1
\end{array}\right]\left[\begin{array}{c}
1 \\
b_{t}(i)
\end{array}\right]+\left[\begin{array}{c}
0 \\
\epsilon_{b, t+1}
\end{array}\right] \\
& H=\left[\begin{array}{cc}
0 & \rho f_{t-1} \\
f_{t-1} & 0
\end{array}\right], \quad R=\operatorname{cov}\left[\begin{array}{c}
0 \\
\epsilon_{b, t+1}
\end{array}\right]=\left[\begin{array}{cc}
0 & 0 \\
0 & \sigma_{b}^{2}
\end{array}\right] \\
& \text { and } \Sigma=\operatorname{cov}\left[\begin{array}{c}
\frac{1-\gamma}{1-\gamma \rho} \epsilon_{f, t}+\epsilon_{p, t} \\
\epsilon_{f, t}
\end{array}\right]=\left[\begin{array}{cc}
\left(\frac{1-\gamma}{1-\gamma \rho}\right)^{2} \sigma_{f}^{2}+\sigma_{p}^{2} & \frac{1-\gamma}{1-\gamma \rho} \sigma_{f}^{2} \\
\frac{1-\gamma}{1-\gamma \rho} \sigma_{f}^{2} & \sigma_{f}^{2}
\end{array}\right]
\end{aligned}
$$


We can write the Kalman filter as

$$
\begin{gathered}
y_{t}=\left[\begin{array}{c}
p_{t} \\
f_{t}
\end{array}\right] \text { and } \beta_{t}=\left[\begin{array}{c}
1 \\
b_{t}(i)
\end{array}\right] \\
\beta_{t}=\beta_{t-1}+V_{t-1} H\left(H^{\top} V_{t-1} H+\Sigma\right)^{-1}\left(y_{t}-H^{\prime} \beta_{t-1}\right) \\
V_{t}=V_{t-1}-V_{t-1} H\left(H^{\top} V_{t-1} H+\Sigma\right)^{-1} V_{t-1}+R
\end{gathered}
$$

where superscript ${ }^{\top}$ represent the transpose of the matrix. The actual price at $t$ is

$$
p_{t}=(1-\gamma) f_{t}+\gamma \rho \hat{p}_{t}+\epsilon_{p, t}
$$

and

$$
\hat{p}_{t}=b_{t-1} f_{t}
$$

where $b_{t-1}$ is the estimate of the coefficient based upon information at $t-1$. It is important to note that this formula of the actual law is derived under the assumption that the decision maker's model is $\mathcal{M}_{0}$.

The sample path of $b_{t}$ induced by Kalman filter shows a small variation of $b_{t}$, converging to the equilibrium value. Consequently, the sample path of $p_{t}$ does not generate sufficient amount of persistent volatility to explain the stylized fact. Evidently, the recursively learning model based upon $\mathcal{M}_{0}$ cannot generate sufficiently large volatility to fit the data with a set of reasonable values of parameters, as the sample path converges to the equilibrium path.

2.3. Learning with a wrong model. As an intermediate step, let us assume for a moment that the decision maker's belief is concentrated at

$$
\mathcal{M}_{1}: \sigma_{b}^{2}>0 .
$$

This is a 'wrong model', in the sense that the rational expectations equilibrium is not an element of $\mathcal{M}_{1}$. The decision maker believes that the parameter of the model is evolving according to a random walk, while the true (unknown) parameter is a constant.

To differentiate the variables induced under $\mathcal{M}_{1}$ from those induced under $\mathcal{M}_{0}$, let us add $i \in\{0,1\}$ to the argument. For example, $\beta_{t}(i)$ is the estimate of $\beta$ based on information available at $t$, if the decision maker's belief is concentrated at $i$.

If the decision maker's belief is concentrated at $\mathcal{M}_{1}$, then we can continue to use the same Kalman filter but the covariance matrix $V_{t}$ of $\beta_{t}$ conditioned on information at $t$ does not vanish. In particular,

$$
\mathrm{E}_{t}\left(b_{t+1}-b_{t}\right)^{2}
$$

is believed to be uniformly bounded away from 0 by the decision maker, because of $\sigma_{b}^{2}>0$. An important question is whether his belief can be self-confirmed by the observations, even though $\sigma_{b}^{2}=0$ in the rational expectations equilibrium.

Proposition 2.1. $b_{t}$ converges to the rational expectations equilibrium weakly, if $\sigma_{b}^{2} \rightarrow 0$.

Despite the fact that $\mathcal{M}_{1}$ does not contain the rational expectations, i.e.,

$$
b=\frac{1-\gamma}{1-\rho \gamma}
$$


the decision maker's belief that $b_{t}$ is evolving according to a random walk is self-confirmed. If so, the wrong model should remain persistent. Since $\sigma_{b}^{2}>0$ and therefore, $V_{t}$ does not vanish, the dynamics of $\beta_{t}$ induced by Kalamn filter can be represented by a constant gain algorithm. For small $\sigma_{b}^{2}>0$, the dynamics of $\beta_{t}$ can be approximated by the solution of stochastic differential equation

$$
d b(\tau)=\bar{b} \tau d \tau+\sqrt{\varepsilon\left(\sigma_{b}^{2}\right)} d W(\tau)
$$

where $\dot{b}=\bar{b}$ is the associated ordinary differential equation of $b_{t}, d W(\tau)$ is the standard Wiener process and $\varepsilon\left(\sigma_{b}^{2}\right)$ is a positive increasing function of $\sigma_{b}^{2}$, which vanishes as $\sigma_{b}^{2} \rightarrow 0$.

For a large $\tau, b_{t}$ stays in a small neighborhood of the stable outcome, which is precisely the rational expectations equilibrium. Thus, $\bar{b} \tau \simeq 0$. Then, $d b(\tau) \simeq \sqrt{\varepsilon\left(\sigma_{b}^{2}\right)} d W(\tau)$, implying that the evolution of $b_{t}$ is almost a random walk. Since $b_{t}$ stays in the small neighborhood of the stable stationary point of the mean dynamics with probability close to 1 , the decision maker observes that $b_{t}$ evolves according to a random walk.

Since the decision maker believes that $b_{t}$ evolves according to a random walk, the Kalman filter algorithm can be approximated by a stochastic differential equation, under which $b_{t}$ evolves almost according to a random walk. Because he believes a random walk of $b_{t}$ (which is not true), the decision maker observes the random walk of $b_{t}$ induced by his learning behavior. Unless $b_{t}$ moves away from the stable stationary point, which occurs rarely, the decision maker can detect his belief is incorrect only with vanishingly small probability. This is why a wrong belief can be persistent. ${ }^{3}$

It has been known that a wrong belief can be persistent, if the gain of truth assumption fails (Esponda and Pouzo (2014)). One might argue that the persistence of $\mathcal{M}_{1}$ is a direct consequence of the fact that the belief of the decision maker fails to satisfy the grain of truth assumption (Kalai and Lehrer (1993)).

We claim otherwise, to demonstrate that the problem arising from the presence of a wrong model can be insidious. We modify this example in the same way as Evans, Honkapohja, Sargent, and Williams (2013)), to show that the persistence of a wrong belief survives the grain of truth assumption, but also the learning under the true model is "disrupted" by the presence of the wrong belief.

2.4. Two competing models. Imagine a policy maker who relies on forecasts from various sources. Being aware that every forecast can be off the mark, the policy maker combines different forecasts to form his own forecast which is then used to generate the data. Let us assume that there are three players: two forecasters indexed by $i \in\{0,1\}$ and one decision maker. Forecaster $i$ believes in $\mathcal{M}_{i}$, and uses the Kalman filter to compute $\beta_{t}(i)=\left(1, b_{t}(i)^{\top}\right.$ and generate forecast

$$
\hat{p}_{t}(i)=\beta_{t-1}(i) f_{t}
$$

Upon receiving the two forecasts, the decision maker choose the weight $\pi_{t-1}$ to form a forecast

$$
\hat{p}_{t}=\left(1-\pi_{t-1}\right) \hat{p}_{t}(0)+\pi_{t-1} \hat{p}_{t}(1)
$$

\footnotetext{
${ }^{3}$ Using the language of Hansen and Sargent (2007), the detection error probability is so large that the decision maker cannot differentiate the wrong model from the true model.
} 
The actual law of motion is then

$$
p_{t}=(1-\gamma) f_{t}+\gamma \mathrm{E}_{t} f_{t+1}+\epsilon_{p, t}=(1-\gamma) f_{t}+\gamma \rho \hat{p}_{t}+\epsilon_{p, t} .
$$

The decision maker is also Bayesian who then updates $\pi_{t}$ according to Bayes rule, from given prior $\pi_{0} \in(0,1)$. Since $\pi_{0}>0$, the decision maker assigns a positive weight to the true model so that his belief satisfies the gain of truth assumption.

Since $\mathcal{M}_{1}$ is assigned a positive probability which is a wrong model, the model of the decision maker is a wrong model, or misspecified. The model is misspecified, not because it misses a variable in the true model but because it contains a variable which is not in the true model.

If the data generating process is independent of the actions of the decision maker, then this sort of practice of starting from a larger model is rather innocuous. The data will show that the variables not in the true model is insignificant. We shall show otherwise, if the data generating process is endogenous.

The simulations of the dynamic system indicate that $\pi_{t} \rightarrow 0$ with a positive probability, but also $\pi_{t} \rightarrow 1$ with a positive probability. This result is reminiscent to the observations by Evans, Honkapohja, Sargent, and Williams (2013). In fact, the principal investigator has shown that the dynamic system has now two locally stable points.

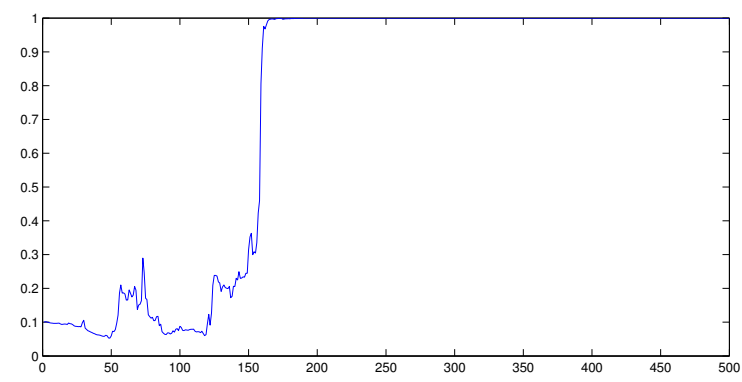

FiguRE 1. Convergence to $\mathcal{M}_{1}$. Note that after reaching $\pi_{t}=1, \pi_{t}$ does not move away from its neighborhood for a long time.

Proposition 2.2. $\pi_{t}=0$ and $\pi_{t}=1$ are two locally stable points.

At first, this result appears to be contradicting Kalai and Lehrer (1993), since the decision maker's belief may converge to a wrong model $\left(\pi_{t}=1\right)$ with a positive probability, despite the fact that his prior satisfies the grain of truth assumption, as it assigns a positive weight to the true model $\mathcal{M}_{0}$.

The key observation is that $b_{t}(1)$ and $b_{t}(0)$ are evolving according to two different time scales. Since $b_{t}(0)$ evolves at the rate of $1 / t$, while $b_{t}(1)$ evolves at a rate uniformly bounded away from $0, b_{t}(1)$ evolves at the "infinitely" faster rate than $b_{t}(0)$ in the limit. Based upon the fact that the odd ratio $\left(1-\pi_{t}\right) / \pi_{t}$ is equal to the product of the likelihood ratio up to time $t$, we can show that $\pi_{t}$ evolves at the same rate as $1 / t$.

We invoke two time scale stochastic approximation technique (Borkar (2008)). For a fixed pair $\left(b_{t}(0), \pi_{t}\right)=(b(0), \pi)$, we approximate the stationary distribution of $b_{t}(1)$. Then, assuming that $b_{t}(1)$ is realized according to the stationary distribution in the limit, we examine the asymptotic properties of $\left(b_{t}(0), \pi_{t}\right)$ as a limit of a decreasing gain formula. 


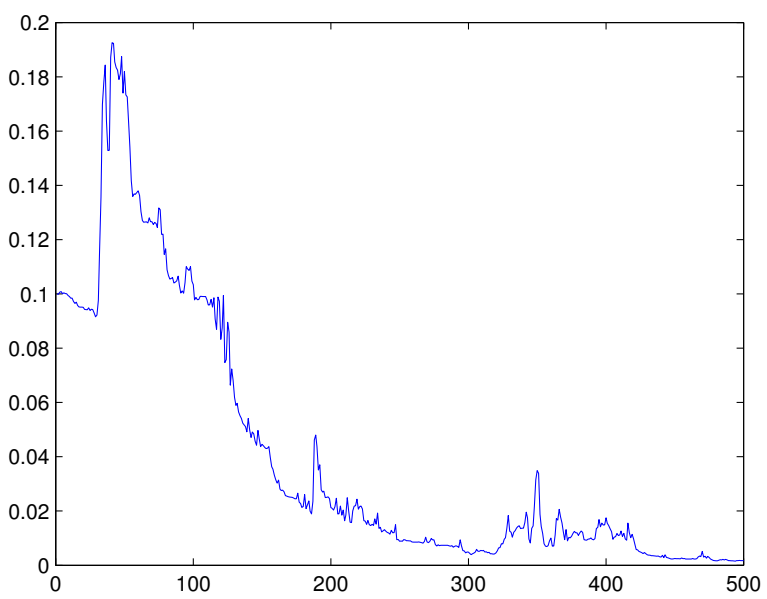

FiguRE 2. Convergence to $\mathcal{M}_{0}$. Even after $\pi_{t}$ enters a small neighborhood of locally stable point $\pi_{t}=0, \pi_{t}$ tends to move away occasionally. Note that the scale of vertical axis, which ranges between 0 and 0.2 (instead of 1 ).

Although $\mathcal{M}_{1}$ is a wrong belief, the learning behavior induced by $\mathcal{M}_{1}$ has an edge over the learning behavior under true model $\mathcal{M}_{0}$. Under $\mathcal{M}_{1}$, the Kalman filter updates much more quickly, which helps to reduce the forecasting error significantly better than under $\mathcal{M}_{0}$. Because the fast adjustment rate of Kalman filter under $\mathcal{M}_{1}$, the decision maker ends up with using $\mathcal{M}_{1}$ over the set of sample paths with a positive probability.

Under the Gaussian assumptions, we can calculate the set of parameter values, which lead to either locally stable point, and the basin of attraction of either stable point has non-empty interior.

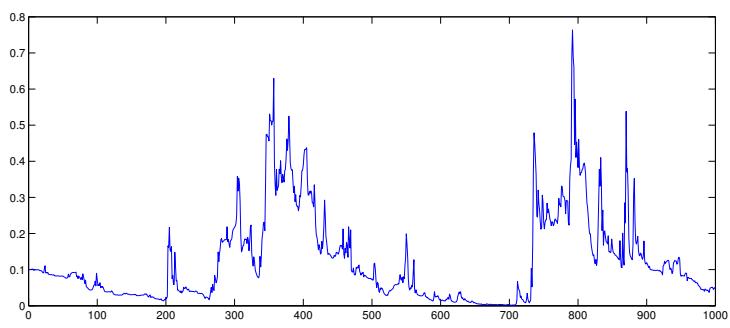

FIGURE 3. The spike can reach as high as 0.8 , and is recurrent. Still, the sample path is moving back toward $\pi_{t}=0$.

Figure 3 reports more intriguing sample path. This sample path converges to $\pi_{t}=$ 0 . But, while the sample path of $\pi_{t}$ stays in a neighborhood of $\pi_{t}=0$, it experiences occasional long excursion from the stable point, often approaching to the neighborhood of $\pi_{t}=1$. Our task is to explain whether this episode is a systematic part of the dynamic system of $\left(\beta_{t}(0), \beta_{t}(1), \pi_{t}\right)$, and if so, our next question is how to explain this recurrent 
dynamics away from the stable point. A related point is that $\pi_{t}$ appears to be significantly more "stable" around $\pi_{t}=1$, than in the neighborhood of $\pi_{t}=0$.

This is quite pessimistic conclusion. If a wrong model $\left(\mathcal{M}_{1}\right)$ is available to a decision maker, and if the decision maker uses the wrong model to influence the actual law of motion, the decision maker ends up with believing in the wrong model, but also will spend most of time in doing so. Even though $\pi_{t}=0$ is a locally stable point where the decision maker can learn to play a rational expectations equilibrium, he behaves as if $\mathcal{M}_{1}$ is the true model. The resulting dynamics of stat variable will entail significantly more volatility than along the rational expectation equilibrium path.

It is true that if the decision maker's belief converges to $\mathcal{M}_{0}$ and therefore, learns to play the rational expectations equilibrium, $\pi_{t}$ will stay in a small neighborhood of $\pi_{t}=0$. However, even in the neighborhood of $\pi_{t}=0$, the dynamics of $\pi_{t}$ appears to deviate significantly from the rational expectations equilibrium path, as Figure 3 indicates.

Conjecture 2.3. Even if $\pi_{t}$ is in the neighborhood of another locally stable point $\pi_{t}=0$, the sample path entails recurrent spikes, excess volatility and serial correlation (after detrending the real GDP), which is not predicted by the rational expectations equilibrium.

This excess volatility is due to the fact that $\beta_{t}(1)$ responds to much more quickly to any significant amount of forecasting errors. Since the event that $\beta_{t}(0)$ generates a significant amount of forecasting errors in the neighborhood of $\pi_{t}=0$ is a rare event, the spike occur infrequently, albeit recurrent, as illustrated by Figure 3. Since the spike occurs regularly, the data appears to show serial correlation of $\pi_{t}$.

To understand the mechanism behind the dynamics of Figure 3, suppose that $\beta_{t}(0)=$ $(1-\gamma) /(1-\gamma \rho)$. We can show that $\beta_{t}(1)=(1-\gamma) /(1-\gamma \rho)$ in the neighborhood of $\pi_{t}=0$. Since $\beta_{t}(0)$ converges to $(1-\gamma) /(1-\gamma \rho)$ with probability 1 , almost all sample paths of $\beta_{t}(0)$ stay within a small neighborhood of $(1-\gamma) /(1-\gamma \rho)$, but with a vanishingly small probability, some sample paths visit outside of the small neighborhood. Along the equilibrium path, the mean squared error of $\mathcal{M}_{0}$ remains within a small neighborhood of the minimal mean squared forecasting error with probability 1 . The mean squared error is so small that the decision maker uses the prediction from $\mathcal{M}_{0}$ almost always.

Consider a sequence of $\left(\epsilon_{s, t}, \epsilon_{f, t}\right)$ which pushes the actual exchange rate $s_{t}$ so that the forecasting error of $\mathcal{M}_{0}$ is larger than a small threshold. Recall that the mean forecast from $\mathcal{M}_{1}$ is the same as the mean forecast from $\mathcal{M}_{0}$, but is only noisier. Upon observing a larger than usual forecasting error, both $\mathcal{M}_{0}$ and $\mathcal{M}_{1}$ updates the forecast by adjusting $\beta_{t}(0)$ and $\beta_{t}(1)$.

Since $\beta_{t}(1)$ adjusts itself much faster than $\beta_{t}(0)$, the forecasting error of $\mathcal{M}_{1}$ quickly becomes smaller than the forecasting error of $\mathcal{M}_{0}$. As a result, the forecast of $\mathcal{M}_{1}$ is assigned more weight quickly, which results in a sharp increase of $\pi_{t}$. As the prediction of $\mathcal{M}_{1}$ is used with a larger weight, the actual exchange rate $s_{t}$ starts to move to the same direction as the initial exchange which deviates from the forecast. Again, $\beta_{t}(1)$ quickly changes so that its forecast $\beta_{t}(1) f_{t}$ catches up the forecasting error, while $\beta_{t}(0) f_{t}$ moves slowly to the same direction. For large $t$, the rate of change of $\beta_{t}(1)$ is almost infinitely larger than the rate of change of $\beta_{1}(0)$. While this feedback between the change of $\beta_{t}(1)$ and $s_{t}$ continues, the weight assigned to $\mathcal{M}_{1}$ increases almost discontinuously. 
This feedback process slows down as $\pi_{t}$ approaches 1 , and is eventually taken over by the mean dynamics. Since the state is in the basin of attraction for $\pi_{t}=0$, the mean dynamics pushes $\pi_{t}$ toward the locally stable point. This observation explains why the spike in Figure fg: convergence to 0 ldp is asymmetric: $\pi_{t}$ practically jumps up, and then, gradually moves down to the locally stable point.

The main task of the proposed project is to formalize the intuition, by analyzing the large deviation properties of the learning dynamics. The key is to analyze the large deviation properties of the dynamic system, around the two locally stable points. Based upon the observations of simulations, we have a number of plausible conjectures.

Conjecture 2.4. The large deviation rate function around $\pi_{t}=1$ is larger than that in the neighborhood of $\pi_{t}=0$. The large deviation rate function is smaller around the locally stable point $\pi_{t}=0$, when $\mathcal{M}_{1}$ is present than otherwise.

Since it is significantly more difficult to escape from the neighborhood of $\pi_{t}=1$ than from the neighborhood of $\pi_{t}=0$, the dynamic system is exponentially longer time in the neighborhood of $\pi_{t}=1$. The presence of the alternative model $\mathcal{M}_{1}$ makes it easier for the endogenous variables to escape from the stable point, generating more volatile sample path. Repeated long simulations will be needed to identify the large deviation properties along the sample paths.

2.5. Sample path properties. Cho, Williams, and Sargent (2002) demonstrated that the large deviation property of the learning dynamics is the key to understand the global dynamics of the Phillips curve example of Sargent (1999). The mechanism of the escape dynamics in the present example is different in an important way from what we found in Sargent (1999). Understanding the mechanism of escape will offer a new way of building a model to generate interesting dynamics.

The escape in Sargent (1999) is triggered by the unintentional experiments by the decision maker, which leads to the discovery of the true model during the episode of escape away from the stable point. During the escape from the stable self-confirming equilibrium in Sargent (1999), the decision maker behaves as if he discovers the true model. In the present example, the escape is triggered by the rapid adjustment of the wrong model, which re-enforces the belief that the wrong model is closer to the true model. During the escape, the decision maker assigns a larger weight to a wrong model.

Understanding the large deviation properties and the global dynamics of the present model provides a useful set of tools to explain a number of puzzles in the financial markets, which cannot be easily explained by the dynamics of fundamentals.

2.5.1. Excess volatility. The volatility in some markets, such as the exchange market, is known to far exceed what the prediction of equilibrium models, or the level implied by the volatility of the real sector. Our approach provides a parsimonious way to explaining the volatility, which does not appear to be linked to the fundamentals of economy. Some papers tried to explain the excess volatility through bounded rationality, such as the learning behavior of the agents. The key weakness of the existing approach is that the extra source of uncertainty was imposed by the modeler, without any explanation for the reason why the decision maker has to maintain the behavior, or continue to entertain the 
perceive model. Our approach is significantly more disciplined, as we require that the perceived model, albeit wrong, be confirmed by the data.

2.5.2. Recurrent spikes. Bubbles arise and burst recurrently, and financial crises occur repeatedly, often without cyclical behavior in fundamentals. One way is to introduce extraneous state variables such as sunspots to generate multiple equilibria, and construct another equilibrium which involves regime switches, to explain recurrent spikes. This line of method admits other equilibria without recurrent spikes. Our approach generates a sharper prediction, allowing to examine how the exogenous parameters of the economy influences the dynamics. In particular, the large deviation rate function tells us the frequency of the recurrent spikes and other useful information.

2.5.3. Asymmetric dynamics. Bubbles form over many periods, but burst over short periods. Financial crises are characterized by sharp devaluation of the home currency, followed by gradual recovery. The asymmetry of dynamics poses considerable challenge. Our approach provides a simple framework to generate asymmetric dynamics. The escape from the self-confirming equilibrium should be sudden, because the escape is triggered by the event which prompts the decision maker to use the forecasting rule which adjust much more quickly than the decreasing gain algorithm. The recovery should be gradual, as the endogenous variables move around the trajectory of the associate ordinary differential equation.

2.5.4. Testable implications. The calculation of the large deviation allows us to compute the probability and the frequency of the sharp changes. We can derive testable implications of the escapes about their direction, size and frequency. Due to the non-linearity of Kalaman filter, we may have to rely on numerical analysis to calculate the key large deviation parameters. 


\section{REFERENCES}

Bacchetta, P., And E. van Wincoop (2013): "On the Unstable Relationship Between Exchange Rates and Macroeconomic Fundamentals," Journal of International Economics, 91, 18-26.

Benhabib, J., And C. Dave (2014): "Learning, Large Deviations, and Rare Events," Review of Economic Dynamics, 17, 367-82.

Borkar, V. S. (2008): Stochastic Approximation: A Dynamical Systems Viewpoint. Cambridge University Press.

Cho, I.-K., N. Williams, And T. J. Sargent (2002): "Escaping Nash Inflation," Review of Economic Studies, 69, 1-40.

Cogley, T., And T. J. Sargent (2005): "Drift and Volatilities: Monetary Policies and Outcomes in the post WWII US," Review of Economic Dynamics, 8, 262-302.

Esponda, I., AND D. Pouzo (2014): "An Equilibrium Framework for Players with Misspecified Models," University of Washington and University of California, Berkeley.

Evans, G., S. Honkapohja, T. Sargent, And N. Williams (2013): "Bayesian Model Averaging, Learning and Model Selection," in Macroeconomic at the Service of Public Policy, ed. by T. J. Sargent, and J. Vilmunen, pp. 99-119. Oxford University Press.

FARMer, R. (1993): The Macroeconomics of Self-Fulfilling Prophecies. MIT Press.

- (2010): Expectations, Employment and Prices. Oxford University Press.

Fernandez-Villaverde, J., AND J. Rubio-Ramirez (2007): "How Structural are Structural Parameters?," in NBER Macroeconomics Annual. MIT Press.

Hansen, L. P., And T. J. Sargent (2007): Robustness. Princeton University Press.

Inoue, A., AND B. Rossi (2011): "Identifying the Sources of Instabilities in Macroeconomic Fluctuations," The Review of Economics and Statistics, 93, 1186-1204.

KAlai, E., AND E. Lehrer (1993): "Rational Learning Leads to Nash Equilibrium," Econometrica, 61, 1019-1045.

LucAs, JR., R. E. (1976): "Econometric Policy Evaluation: A Critique," in The Phillips Curve and Labor Markets, ed. by K. Brunner, and A. Meltzer. Carnegie-Rochester Conf. Series on Public Policy.

Marcet, A., AND J. P. Nicolini (2003): "Recurrent Hyperinflations and Learning," American Economic Review, 93, 1476-98.

SARgent, T. J. (1999): The Conquest of American Inflation. Princeton University Press.

Sargent, T. J., AND N. Williams (2005): "Impacts of Priors on Convergence and Escapes from Nash Inflation," Review of Economic Dynamics, 8, 360-91.

Shiller, R. J. (1989): Market Volatility. MIT Press.

Timmermann, A. G. (1996): "Excessive Volatility and Predictability of Stock Prices in Autoregressive Dividend Models with Learning," Review of Economic Studies, 63, 523-57. 